\title{
Endoleak detection using single-acquisition split-bolus dual-energy computer tomography (DECT)
}

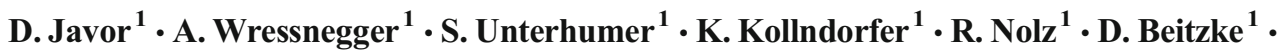 \\ C. Loewe ${ }^{1}$
}

Received: 29 January 2016 / Revised: 19 June 2016 / Accepted: 21 June 2016 /Published online: 19 July 2016

(C) The Author(s) 2016. This article is published with open access at Springerlink.com

\begin{abstract}
Objectives To assess a single-phase, dual-energy computed tomography (DECT) with a split-bolus technique and reconstruction of virtual non-enhanced images for the detection of endoleaks after endovascular aneurysm repair (EVAR).

Methods Fifty patients referred for routine follow-up postEVAR CT and a history of at least one post-EVAR followup CT examination using our standard biphasic (arterial and venous phase) routine protocol (which was used as the reference standard) were included in this prospective trial. An in-patient comparison and an analysis of the splitbolus protocol and the previously used double-phase protocol were performed with regard to differences in diagnostic accuracy, radiation dose, and image quality.

Results The analysis showed a significant reduction of radiation dose of up to $42 \%$, using the single-acquisition splitbolus protocol, while maintaining a comparable diagnostic accuracy (primary endoleak detection rate of $96 \%$ ). Image quality between the two protocols was comparable and only slightly inferior for the split-bolus scan (2.5 vs. 2.4).

Conclusions Using the single-acquisition, split-bolus approach allows for a significant dose reduction while maintaining high image quality, resulting in effective endoleak identification.

Key Points

- A single-acquisition, split-bolus approach allows for a significant dose reduction.
\end{abstract}

D. Javor

domagoj.javor@meduniwien.ac.at

1 Department of Biomedical Imaging and Image-guided Therapy, Medical University of Vienna, Währingergürtel 18-20, A-1090 Vienna, Austria
- Endoleak development is the most common complication after endovascular aortic repair (EVAR).

- CT angiography is the imaging modality of choice for aortic aneurysm evaluation.

Keywords Endoleak $\cdot$ Aorta $\cdot$ Aneurysm $\cdot$ Computed tomography $\cdot$ Angiography

\section{Introduction}

Endovascular aneurysm repair (EVAR) is a widely used, minimally invasive technique for the treatment of infrarenal and thoracic aortic aneurysms, and it has become an accepted alternative to open surgery [1,2]. Endoleak development - defined as persistent periprosthetic flowis the most common complication after EVAR (rate, $16 \%-33 \%[3,4])$ and represents the major risk factor for late rupture of the aneurysm and the main indication for conversion to open repair [5]. It has, therefore, become critical to identify endoleaks that necessitate secondary interventions to ensure long-term success after the procedure and to prevent late rupture. This requires a life-long surveillance strategy with a regular schedule of yearly follow-up examinations.

$\mathrm{CT}$ angiography is considered the imaging modality of choice for pre- and postoperative imaging evaluation of abdominal aortic aneurysms. However, there is an on-going debate about the optimal $\mathrm{CT}$ acquisition protocol to avoid high cumulative radiation doses [6]. Arterial and venous delayed phases are considered necessary because of the variable flow velocities of endoleaks [7-9]. In addition, unenhanced images are useful in differentiating calcifications in the aneurysm sac from periprosthetic contrast enhancement. Recent studies have shown an increased risk of radiation-induced cancer after 
repeated exposure from CT scans [10-14], underlining the need for dose-reduction strategies, especially in patients who require repeated CT scanning. Thus, given the need for lifelong CT follow-up after abdominal stent-graft placement, the standard CT acquisition protocol after EVAR is currently a trade-off between image quality and radiation exposure.

In this prospective trial, we created a single-phase, dualenergy $\mathrm{CT}$ angiography with a split-bolus technique. By injecting intravenous contrast material in two sequential boluses separated by an appropriate time delay, imaging during synchronous arterial and venous delayed phases is possible within one single scan acquisition. If other technical factors are held constant, this approach should reduce the effective radiation dose associated with the CTA examination simply by reducing the number of $\mathrm{CT}$ acquisitions obtained.

Furthermore, dual-energy CT provides a wide range of post-processing capabilities for the evaluation of CT angiography data sets $[15,16]$, and, by taking advantage of one of the main features of dual-energy CT- the possibility to calculate virtual unenhanced images - a further radiation dose reduction should be possible by replacing standard unenhanced images with virtual unenhanced images.

Thus, the aim of this study was to assess whether the image quality and endoleak diagnosis remained unchanged when applying a modified CT acquisition protocol with reduced radiation exposure compared to the previously used standard biphasic protocol (baseline scan).

\section{Materials and methods}

\section{Patients}

Fifty consecutive patients referred to our department to undergo CT angiography after EVAR of abdominal aortic aneurysms were prospectively enrolled in this study. Only patients with a history of at least 6 months poststent-graft implantation and at least one prior post-EVAR follow-up CT examination at our institution, using the standard biphasic routine protocol (baseline scan), were enrolled (follow-up protocol after EVAR was a CT at 3 days, 6 months, and 12 months, and yearly thereafter). Exclusion criteria included contraindication to intravenous administration of iodine contrast medium, hyperthyroidism, and the presence of renal failure.

After the conclusion of the study, five of the patients were treated because of type II endoleaks with growing aneurysm sacs and type I endoleaks.

The study plan is described in Fig. 2, and the stent-graft devices used for EVAR are listed in Table 1.

Written informed consent was obtained from all study patients prior to inclusion in the study. The study was approved by the institutional review board (Ek-Nr 2162/2013).
Table 1 Stent-graft devices used for EVAR

Stentgraft

Medtronic Endurant II 17

Gore Excluder $\quad 13$

Cook Zenith 5

Medtronic Talent 3

Trivascular Ovation $\quad 2$

Bolton Treovance $\quad 2$

Vascutec Anaconda $\quad 2$

Vanguard 2

Jotec E-Vita 1

Unknown (due to EVAR in external hospitals) 3

\section{CT acquisition protocol (standard biphasic baseline scan)}

All examinations were performed using a second-generation, dual-source CT scanner (DSCT-SOMATOM Definition Flash, Siemens).

The protocol included an arterial phase acquisition, followed by a venous phase acquisition limited to the extent of the stent graft.

Dual-source MSCTA was performed after the injection of $110 \mathrm{ml}$ Iomeron 400 at an injection rate of $6 \mathrm{ml} / \mathrm{s}$ and initiated $15 \mathrm{~s}$ after the threshold at the level of the abdominal aorta reached $150 \mathrm{HU}$ (bolus-tracking technique). Venous phase acquisition was initiated $18 \mathrm{~s}$ after the arterial phase. The tube voltage was set to $120 \mathrm{kV}$ with a tube current-time product of $120 \mathrm{mAsref} /$ rot.

\section{CT acquisition protocol (split bolus)}

The split-bolus scan was performed in the caudo-cranial direction with the same range as the standard baseline scan protocol using the dual-source CT scanner described above.

The total contrast medium volume was determined as a function of the patient's BMI, and four subgroups were formed, ranging from $90-130 \mathrm{ml}$ total volume, as illustrated in Table 2.

The first bolus of contrast medium was administered, based on the body mass index of the patient, followed by a chaser bolus of $20 \mathrm{ml}$ saline solution at a $4 \mathrm{ml} / \mathrm{s}$ flow rate. The time between starting the first and the second bolus was $35 \mathrm{~s}$ in all the patients. The bolus split ratio was chosen to be 40:60. The reason for the chosen ratio was to make more contrast medium available for the early and clinically more important type I and type III endoleaks.

The scanning delay was determined using a bolus-tracking technique by placing a region of interest (ROI) at the level of the abdominal aorta and simultaneously starting the dynamic monitoring scan. The trigger threshold inside the ROI was set at an absolute value of $130 \mathrm{HU}$. 
Table 2 Total contrast medium volume and injection flow rates as a function of the patient's BMI subdivided into four subgroups

\begin{tabular}{|c|c|c|c|c|}
\hline & $<20 \mathrm{BMI}$ & 20-25 BMI & 25-30 BMI & $>30 \mathrm{BMI}$ \\
\hline Total contrast medium quantity & $90 \mathrm{ml}$ & $100 \mathrm{ml}$ & $110 \mathrm{ml}$ & $130 \mathrm{ml}$ \\
\hline Contrast medium bolus 1 & $35 \mathrm{ml} 2.3 \mathrm{ml} / \mathrm{s}$ & $40 \mathrm{ml} 2.6 \mathrm{ml} / \mathrm{s}$ & $45 \mathrm{ml} 3 \mathrm{ml} / \mathrm{s}$ & $55 \mathrm{ml} 3.6 \mathrm{ml} / \mathrm{s}$ \\
\hline Contrast medium bolus 2 & $55 \mathrm{ml} 3.6 \mathrm{ml} / \mathrm{s}$ & $60 \mathrm{ml} 4 \mathrm{ml} / \mathrm{s}$ & $65 \mathrm{ml} 4.3 \mathrm{ml} / \mathrm{s}$ & $75 \mathrm{ml} 5 \mathrm{ml} / \mathrm{s}$ \\
\hline
\end{tabular}

A dual-energy scan was initiated $8 \mathrm{~s}$ after the attenuation reached the predefined threshold of $130 \mathrm{HU}$.

The tube voltage for tube A was set to $80 \mathrm{kV}$, with a tube current-time product of $215 \mathrm{mAsref} / \mathrm{rot}$, and the tube voltage for tube B was $140 \mathrm{kV}$, with a tube current-time product of 83 $\mathrm{mAsref} /$ rot. The chosen parameters ensured a similar CT dose index (CTDI) for the dual-energy acquisition compared to the CTDI of the arterial phase of our baseline scan (CTDIvol $=8.11 \mathrm{mGy}$ ). This served to make the DECT scanning protocol radiation-neutral compared to a single-phase arterial scan.

\section{Data processing and evaluation of the split-bolus scan}

From the raw helical projection data of both tubes in the dualenergy acquisition, three separate data sets were generated: 80 $\mathrm{kVp}, 140 \mathrm{kVp}$, and mixed or fused images. From each of these data sets, two series of axial images were then reconstructed. A virtual non-contrast CT data set (VNC) and an iodine colour-coded data set that showed iodine distribution over the virtual non-contrast image were reconstructed transversally and sagittally (Fig. 1).

\section{Radiation dose estimation}

For the baseline scan acquired using the standard protocol, as well as for the current dual-energy scan, the volume CT dose index [CTDI vol (mGy)] and total dose-length product [DLP $(\mathrm{mGy} \times \mathrm{cm})]$ were recorded from the CT dose report and compared.

The obtained values of DLP were converted to a corresponding individual effective radiation dose in millisieverts $(\mathrm{mSv})$ by multiplying the DLP by a conversion coefficient $[\mathrm{k}(\mathrm{mSv} / \mathrm{mGy} \times \mathrm{cm})]$ (normalised effective dose per DLP over various body regions for adults based on a 32-cm-diameter CT body phantom). The CT scans included the abdomen $(\mathrm{k}=0.015 \mathrm{mSv} / \mathrm{mGy} \times \mathrm{cm})$ and, in some cases, the chest $(\mathrm{k}=0.014 \mathrm{mSv} / \mathrm{mGy} \times \mathrm{cm})$; therefore, the mean of both region-specific conversion coefficients, $\mathrm{k}=0.0145 \mathrm{mSv} /$ $\mathrm{mGy} \times \mathrm{cm}$, was used [17-19].

\section{Data Interpretation: reading sessions}

The cases were evaluated with regard to endoleak diagnosis and image quality. Two radiologists with 11 and 6 years of experience in body CT angiography, who were blinded to the patient's clinical information and previous imaging findings, evaluated the images.

Axial images, as well as multiplanar reconstructions, were reviewed on external workstations after anonymisation and randomisation.

Two different reading sessions were performed by each reader, separately, and the results were compared subsequently. In cases of disagreement between the two readers, the final decision was made by consensus.

In the first session (session A), the baseline scan was evaluated. The information obtained by the reading of these cases was considered the reference standard for the diagnosis of an endoleak.

In the second session (session B), the readers evaluated the new single-acquisition, dual-energy image data sets. The interval between reading sessions A and B was 3 weeks.

Each case was assessed for the presence of an endoleak. In this evaluation, endoleaks were classified as previously described [20].

Furthermore, the maximum diameter of the aneurysm sac was measured and a potential aneurysm expansion or reduction was assessed. All three image planes (and curved MPRs) were analysed to evaluate the centre line of the aorta. The maximum axial diameter of the aneurysm sac perpendicular to the centre line (longitudinal axis) of the aorta was measured. Aneurysm reduction was defined as a reduction of $\geq 5 \mathrm{~mm}$ in the maximum diameter compared to the previous examination, whereas aneurysm expansion was defined as an increase of $\geq 5 \mathrm{~mm}$ in the maximum diameter. Variations of $<5 \mathrm{~mm}$ diameter were considered not significant, and the aneurysmal sac was then regarded as stable [21].

The two readers evaluated subjective image quality, using a four-point scale [22], as follows: grade 0 (non-diagnostic); grade 1 (poorly diagnostic); grade 2 (good quality); grade 3 (excellent).

In case of conformity between the two examinations regarding the presence or non-presence of an endoleak, the split-bolus acquisition was considered true positive or true negative (primary agreement rate). An additional requirement was that the diameter of the aneurysm sac was constant or decreasing if no endoleak was present in either examination (single-acquisition DE and biphasic).

In case of a discrepancy between the follow-up examination using the baseline scan and the split-bolus acquisition that 
Fig. 1 The first three images show the $80-\mathrm{kVp}, 140-\mathrm{kVp}$, and mixed or fused images. Iodine colour-coded (fourth image) and virtual non-contrast (fifth image) CT data sets are also shown
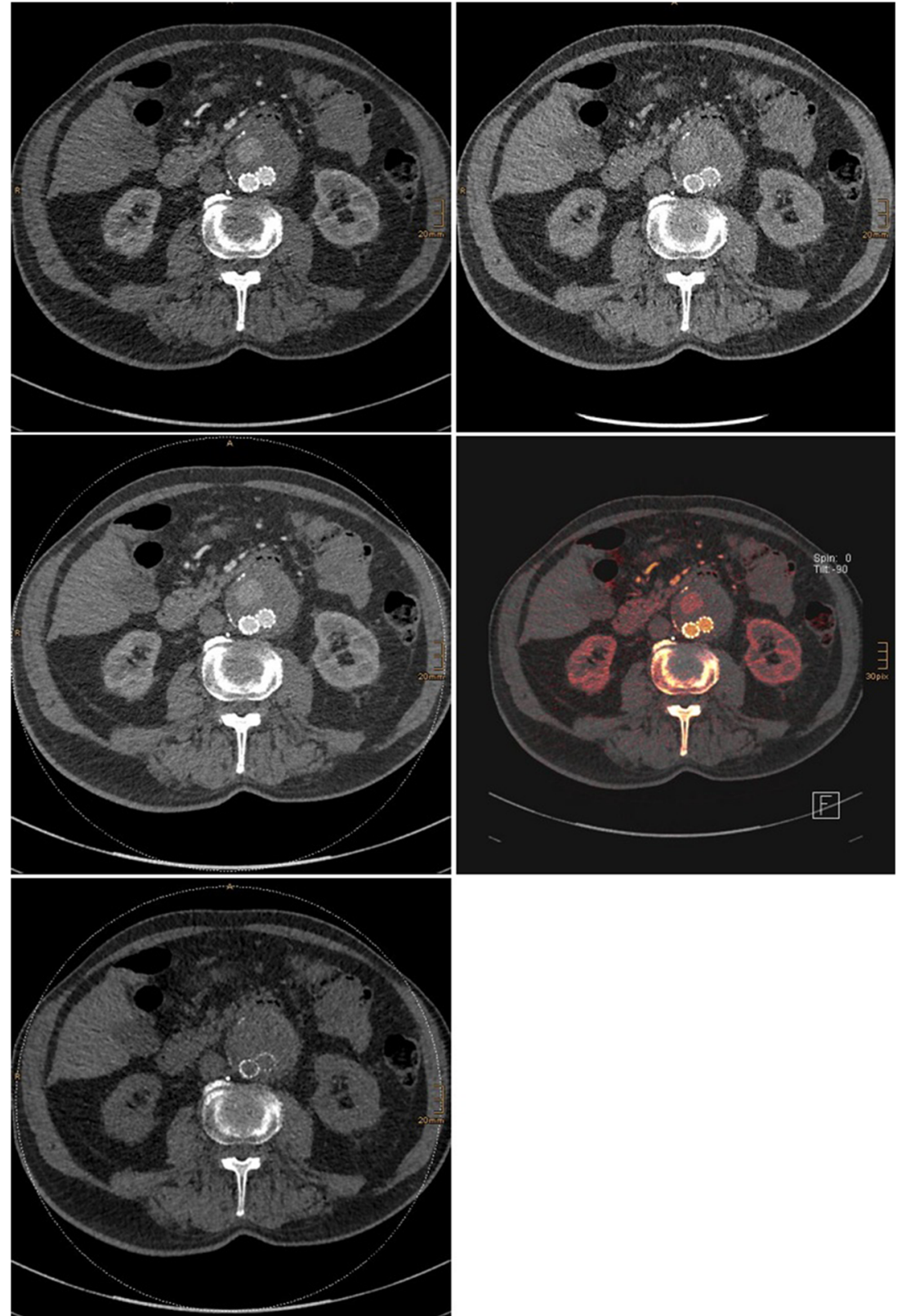

could not be explained by the normal clinical course (e.g., newly developed late-onset endoleak and decreasing/ constant diameter of the aneurysm sac or disappearance of a previously described endoleak and increasing diameter), an additional MRI examination of the aorta was performed as the final reference standard, and the result was defined as the secondary agreement rate. MRI is currently considered the gold standard for endoleak detection and does not further increase the radiation dose to the patient [23]; furthermore, new unenhanced MRI techniques appear to be promising advancements with regard to endoleak detection [24]. The two readers performed an additional reading session to evaluate the MRI results.

\section{MRI angiography protocol}

The patients were examined on a 1.5-T MR scanner (Magnetom Avanto, Siemens) with a gradient amplitude of $40 \mathrm{mT} / \mathrm{m}$ along the $\mathrm{x}$ - and $\mathrm{y}$-axes and $45 \mathrm{mT} / \mathrm{m}$ along the z-axis and a maximum slew rate of $200 \mathrm{mT} / \mathrm{m} / \mathrm{ms}$. A commercially available six-element flexible body coil (Matrix, Siemens Healthcare) was used to image the abdominal aorta.

After the acquisition of a contrast-free mask (coronal T1weighted gradient-echo sequence) of the whole abdominal aorta and the pelvic vessels, contrast-enhanced, first-pass 3D MRA was performed. A biphasic injection protocol was 
applied with an automatic power injector. Gadobenate dimeglumine was injected at a flow rate of $2 \mathrm{ml} / \mathrm{s}$, followed by a $21-\mathrm{ml}$ saline flush at a flow rate of $1.2 \mathrm{ml} / \mathrm{s}$. The amount of gadobenate dimeglumine was calculated according to the following formula: $0.2 \mathrm{ml} \times$ body weight $(\mathrm{kg})$.

MRA in three phases (arterial, late arterial, and venous) was initiated using a bolus-triggering technique.

Furthermore, a T1 VIBE fat-saturated sequence before and after contrast medium administration was acquired.

\section{Statistical analysis}

Data analysis was performed using the SPSS statistical package (SPSS Windows, version 20.0). Statistical power analysis was performed using the statistical program $G^{*}$ Power (http://www.gpower.hhu.de/). For the detection of small to medium effects (Cohen's $d=0.4)$ in a two-tailed t-test for paired samples with $\alpha=0.05$ and an assumed power $(1-\beta)=0.80$, a total sample size of 50 subjects was calculated. In addition to the descriptive evaluation of the study sample, a paired-samples t-test was performed to compare the radiation parameters between the two protocols. A $p$-value of less than $0.05(p<0.05)$ was considered to indicate statistically significant results.

\section{Results}

There was 1 female and 49 male patients; mean age was 75.83 \pm 7.44 years. The mean follow-up period between EVAR and the split-bolus examination was $12.32 \pm 7.76$ months.
Twenty-three endoleaks were found on the prior baseline examination, of which $9 \%(n=2)$ were seen only on the arterial phase, whereas $30 \%(n=7)$ were visible only on the late venous phase. Of the observed endoleaks, $61 \%(n=14)$ were visible on both the arterial and venous phase.

Twenty endoleaks were found using the split-bolus protocol. One endoleak was seen on the baseline scan, but not on the split-bolus scan. This could be explained by the normal clinical course due to the clearly decreasing diameter of the aneurysm sac. In addition, this result was confirmed by a follow-up CT (using the standard biphasic protocol) after 5 months. Thus, the resulting primary rate of agreement (compared to the baseline examination) was $96 \%(n=21)$, and the rate of non-agreement was $4 \%$. In those $4 \%(n=2)$ of cases of non-agreement, an additional MRI examination was performed according to the study design. This MRI examination confirmed the split-bolus protocol results in every single case. Therefore, there was a secondary agreement rate of $100 \%$ (Fig. 2).

As expected, most of the observed endoleaks were type II ( $85 \%$ ), two endoleaks (10\%) were type I, and one endoleak was classified as type III (5\%).

The diameter of the aneurysm sac increased in size or remained constant in all cases where an endoleak persisted (endoleak visible on both the standard examination as well as the split-bolus protocol). In all cases of non-existent endoleak (on both examinations), the aneurysm sac decreased in size or remained constant between the two examinations.

The image quality of the contrast-enhanced images of the split-bolus scan was rated either as grade 2 (good) or grade 3 (excellent) in all cases, resulting in an overall grading of 2.4. In contrast, the image quality of the VNC data was inferior

Fig. 2 Flowchart of study plan

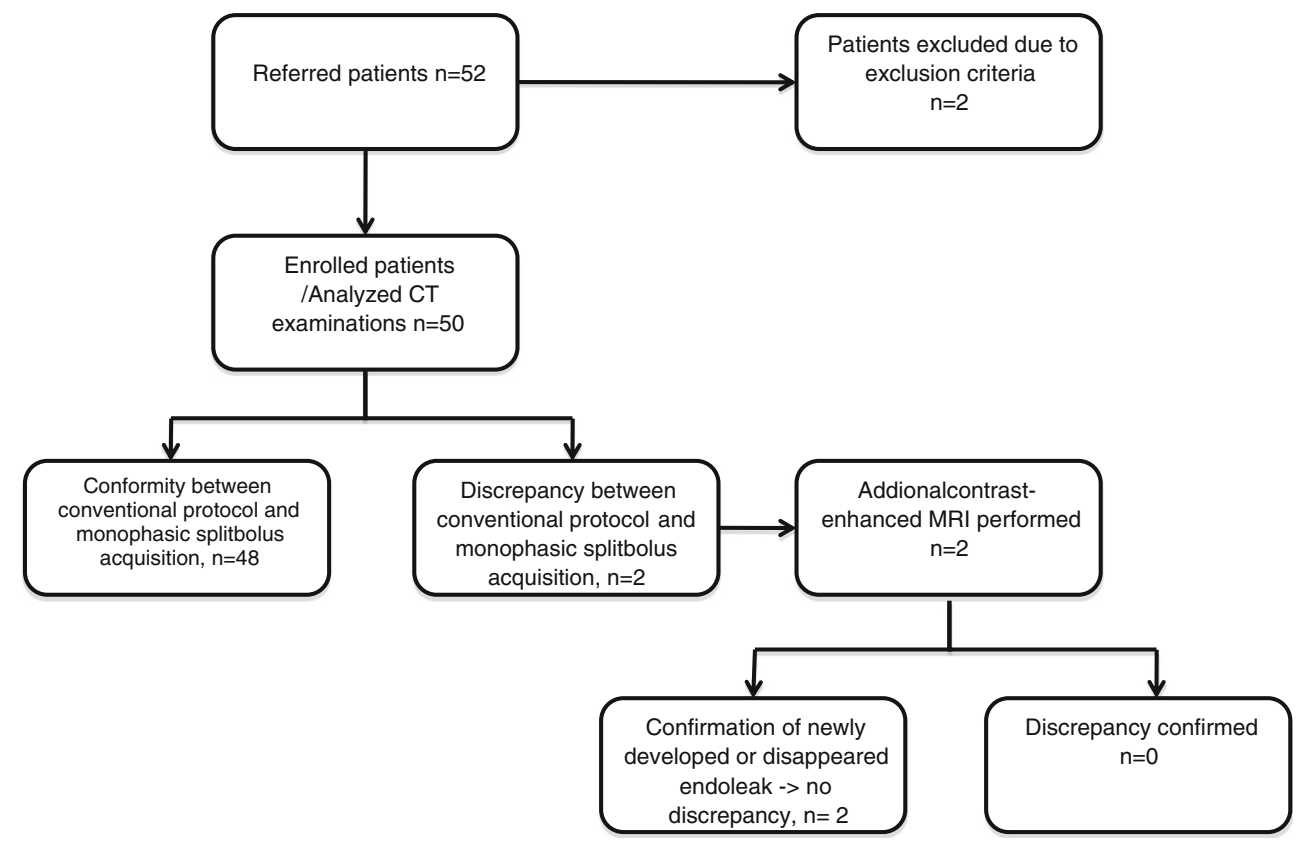


and rated as 1.8. The image quality of the baseline scan was rated 2.5 (contrast-enhanced images) and 2.0 (for native images), respectively.

A paired-samples t-test was conducted to compare radiation dose parameters between the two test protocols. Results revealed a significant difference in the CDTI for the baseline scan $($ mean $=15.4, \mathrm{SD}=5.4)$ and the split-bolus scan $($ mean $=7.9, \mathrm{SD}=1.7 ; \mathrm{t}(46)=-11.17, p<0.001)$. A statistically significant difference was also determined for the total doselength product (DLP). Whereas for the baseline scan a mean DLP of 635.4 mGycm (SD = 270.3) was observed, the mean DLP for the split-bolus scan was $369.9 \mathrm{mGycm}$ [SD =93.0; $\mathrm{t}(46)=-7.83, p<0.001]$. Figure 3 illustrates the relationship of the DLP ( $\mathrm{mGycm}$ ) between the two protocols. The results of this study revealed effective dose values (using a conversion factor of 0.0145 ) of $9.1 \mathrm{mSv}$ for the baseline scan and $5.3 \mathrm{msV}$ for the split-bolus scan.

\section{Discussion}

A single-acquisition, split-bolus protocol performed on a dualenergy CT scanner was used in this study to test the hypothesis that, compared to the conventional biphasic protocol (baseline scan), the total amount of the radiation dose can be reduced while maintaining a comparable endoleak detection rate. As expected, the radiation dose could be reduced significantly when compared to the baseline scan, while the diagnostic accuracy in detecting endoleaks remained unaffected. The obtained values of DLP and the corresponding individual effective radiation dose showed a reduction of $42 \%$.

The detection rate for endoleaks using the singleacquisition protocol was sufficient, with only two cases of discrepancy between the two protocols, which seemingly suggested an apparent minimally reduced detection potential. However, all of those unclear cases were reassessed by obtaining an MRI examination, which confirmed the results of the single-acquisition protocol in every single case, resulting in an overall secondary endoleak detection rate of $100 \%$. The sensitivity for endoleak detection on contrastenhanced MRI has been described as significantly higher compared to that of CT, and MRI is currently the gold standard for endoleak detection $[25,26]$. Based on these findings, the two "discrepant" findings seemed to be attributable more to clinical changes during the follow-up period rather than to
Fig. 3 The diagram illustrates the relationship between the doselength product ( $\mathrm{mGycm}$ ) of the baseline scan and the subsequent split-bolus protocol

\section{DLP Split-Bolus Scan vs. Reference Scan}
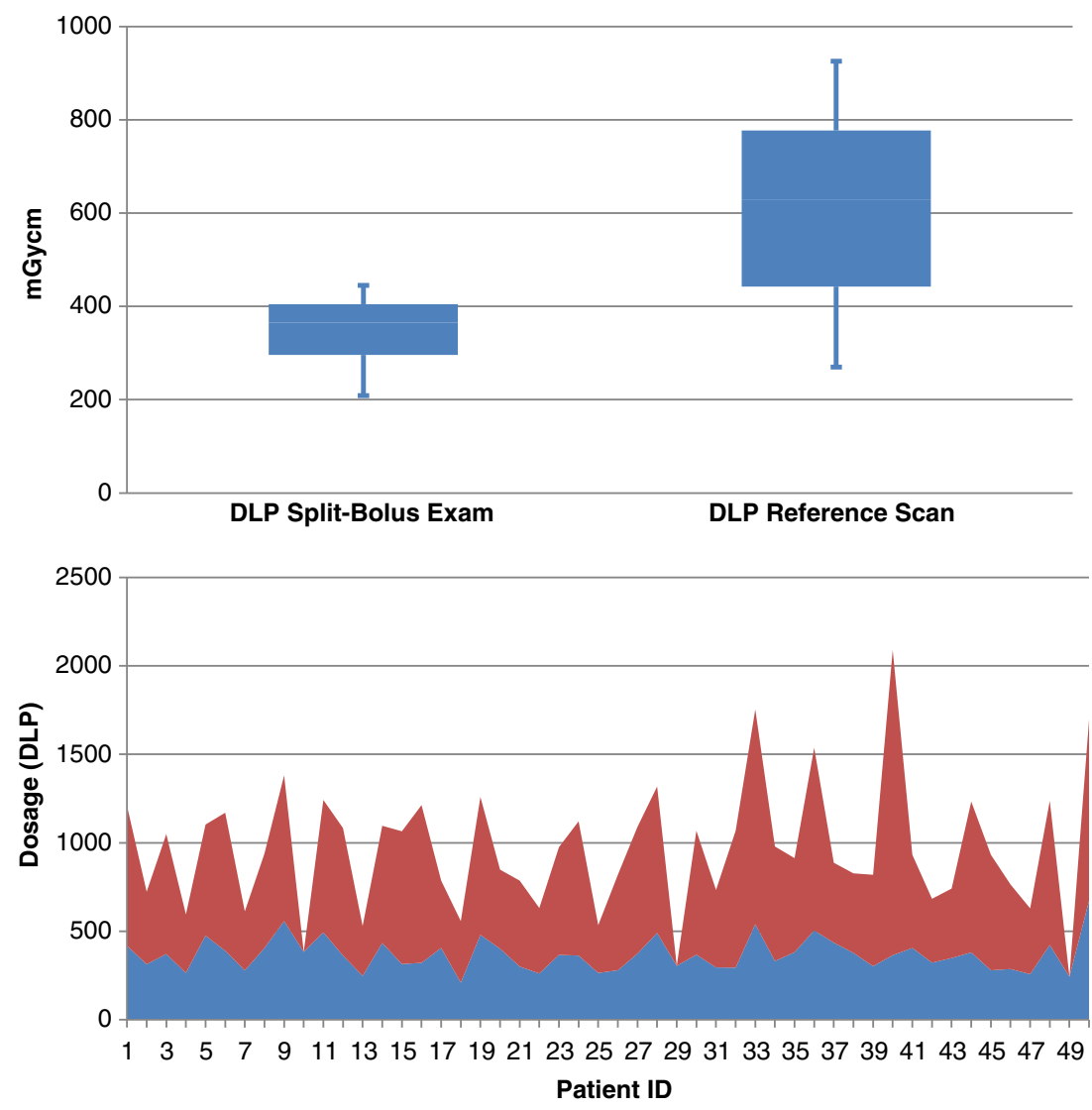

DLP Split-Bolus Exam — DLP Reference Scan 

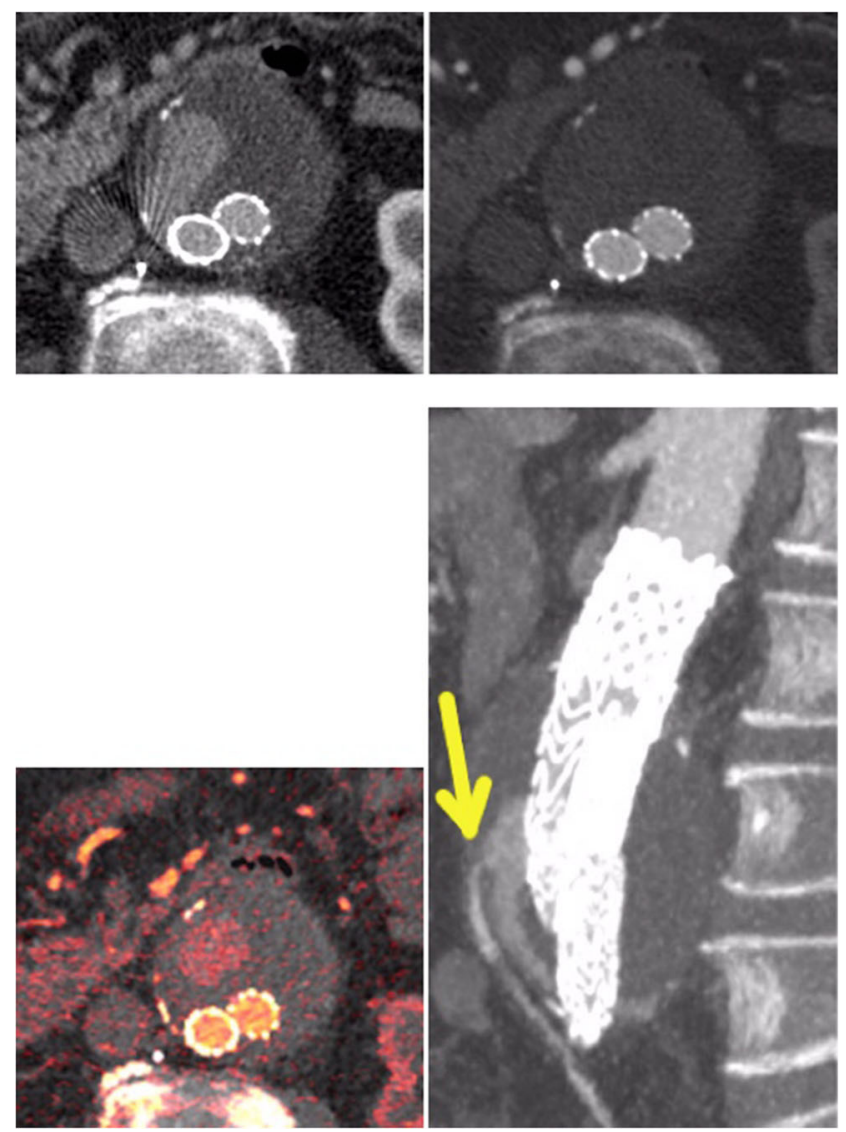

Fig. 4 An example of the usefulness of the split-bolus protocol. On the upper left, an endoleak can be clearly depicted on the late venous phase, whereas on the arterial phase (upper right), the endoleak is hardly visible. The iodine colour-coded data set (lower left, corresponding slice) seems to be particularly favourable for depicting endoleaks. On the lower right (split-bolus, different patient), the endoleak and the feeding artery (inferior mesenteric artery, IMA), are well demarcated

differences in the diagnostic accuracy between the two techniques. The measurements of the aneurysmal sac diameter, which decreased (or remained constant) in the case of non-existent endoleaks and increased in cases of endoleak detection, confirmed our results.

There is an on-going debate about the nature of the ideal CT protocol for endoleak detection. With regard to the ideal bolus timing in contrast-enhanced imaging, it remains, for the time being, somewhat unclear whether an arterial or late venous phase is necessary to detect clinically relevant endoleaks, and various studies recommend one or the other. Since cumulative radiation exposure is an important issue in this group of patients, the number of phases acquired is currently a compromise between diagnostic accuracy and radiation dose.

Some authors advocate a monophasic and some suggest a bi- or even tri-phasic protocol. The reason for this inconclusiveness is that endoleaks have variable flow rates, depending on their source, and the blood pool within the aneurysm sac will, therefore, be detectable at variable time points. As a consequence, optimal bolus timing remains a crucial issue in protocol planning. Late ruptures are very rare in cases of type II endoleaks; therefore, several authors recommend only an arterial phase or even only a native scan for routine follow-up; if the diameter remains unchanged, the risk for rupture should be negligible [27].

Some authors advocate the late venous phase after $100 \mathrm{~s}$ or even after $300 \mathrm{~s}$, arguing that most endoleaks can be detected $[7,8]$. This seems to be true only for type II endoleaks. Highflow type I or type III endoleaks require an arterial phase to be identified [9]. Another role for arterial phase scans is the assessment of aortic branch perfusion (renal, mesenteric, and iliac arteries).

This coincides with our results. Given the large number of type II endoleaks, the evaluation of our results shows that most endoleaks are depictable on both the venous and the arterial phase. However, $30 \%$ of endoleaks were visible exclusively on the venous phase, and two endoleaks (2\%) were seen only on the arterial phase, underlining the need for a combination of arterial and venous phase information for accurate endoleak detection. All of those endoleaks were detectable on the subsequent split-bolus examination (Fig. 4).

We used a split-bolus ratio of 40:60 in order to make more contrast medium available for the early and clinically more important type I and type III endoleaks, but a different ratio of the split bolus could be beneficial, depending on the desired
Fig. 5 An example of the usefulness of the VNC data set. On the left, the contrastenhanced, split-bolus image shows a questionable type II endoleak, possibly arising from a right lumbar artery. On the right, the corresponding slice of the $\mathrm{VNC}$ data set reveals a coarse, low-density calcification at that location
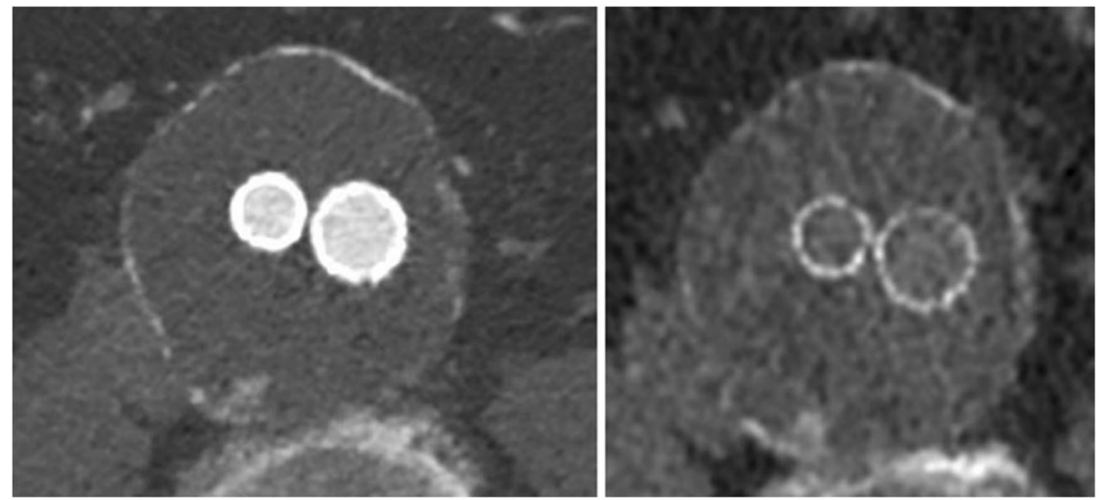
weighting of the arterial or late venous component of the protocol.

One important question is whether non-contrast imaging is necessary. In most cases, calcifications can be distinguished easily from endoleaks on contrast-enhanced CT. However, in a few cases, heavy calcifications, particularly coarse lowdensity calcifications and haematomas, can mimic endoleaks and make diagnosis difficult. The reconstructed virtual noncontrast (VNC) we used facilitated the differentiation between calcifications and endoleaks significantly (Fig. 5). Nevertheless, we can confirm that, compared to non-contrast scans (native phase), VNC data sets have limitations caused by incorrect calcium subtractions and high noise, which lead to poorer image quality [28, 29].

Recently, promising surveillance strategies that involve contrast-enhanced ultrasound (CEUS) have been proposed [30]. This approach reduces the radiation exposure as well as the contrast medium volume injection. These new protocols consist of CT imaging at 1 month and 1 year after treatment, subsequently followed by CEUS. Nevertheless, the limitations of the method must be considered, such as difficulties of visualisations due to obesity, operator dependency, and artefacts due to echo reflection of graft material in the early follow-up period.

However, this study has several limitations. First, the time interval between the two compared examinations (performed using the conventional and then the split-bolus protocol) was, in some cases, rather long ( 3 to 12 months) and it could be argued that a late endoleak could have developed in the interval between the examinations that was not detected by the split-bolus protocol. Although this possibility cannot be entirely excluded, the fact that, in those cases, there was no increase in aneurysm sac diameter supports the validity of the data. Second, the number of cases is rather small. However, power analysis revealed a minimum sample size of 50 patients to detect the expected effects. Nevertheless, larger cohorts may provide a more profound knowledge of potential influencing factors, such as age or gender.

The relatively high endoleak rate in our patient sample can be explained by the fact that patients with endoleaks often require more frequent follow-up examinations, especially when the aneurysm sac is growing or after treatment. This could also explain the high incidence rate of type II endoleaks in our consecutive study population.

In conclusion, a dual-energy, split-bolus protocol appears to be a valid radiation-saving alternative for routine follow-up after EVAR. The comparable endoleak detection rate, as well as the significantly reduced radiation dose, supports this approach.

Acknowledgments Open access funding provided by Medical University of Vienna. The scientific guarantor of this publication is Prof. Dr. Christian Loewe, Medical University Vienna. The authors of this manuscript declare no relationships with any companies, whose products or services may be related to the subject matter of the article. The authors state that this work has not received any funding. Kathrin Kolldorfer kindly provided statistical advice for this manuscript. Methodology: prospective/retrospective, performed at one institution.

Open Access This article is distributed under the terms of the Creative Commons Attribution 4.0 International License (http:// creativecommons.org/licenses/by/4.0/), which permits unrestricted use, distribution, and reproduction in any medium, provided you give appropriate credit to the original author(s) and the source, provide a link to the Creative Commons license, and indicate if changes were made.

\section{References}

1. Greenhalgh RM, Brown LC, Powell JT, Thompson SG, Epstein D, Sculpher MJ (2010) Endovascular versus open repair of abdominal aortic aneurysm. N Engl J Med 362:1863-1871

2. Schermerhorn ML, O'Malley AJ, Jhaveri A, Cotterill P, Pomposelli F, Landon BE (2008) Endovascular vs. open repair of abdominal aortic aneurysms in the Medicare population. $\mathrm{N}$ Engl J Med 358:464-474

3. Hobo R, Buth J (2006) Eurostar CollaboratorsSecondary interventions following endovascular abdominal aortic aneurysm repair using current endografts. A EUROSTAR report. J Vasc Surg 43:896-902

4. Sheehan MK, Ouriel K, Greenberg R et al (2006) Are type II endoleaks after endovascular aneurysm repair endograft dependent? J Vasc Surg 43:657-661

5. Vallabhaneni SR, Harris PL (2001) Lessons learnt from the EUROSTAR registry on endovascular repair of abdominal aneurysm repair. Eur J Radiol 39:34Y41

6. Laks S, Macari M, Chandarana H (2010) Dual-energy computed tomography imaging of the aorta after endovascular repair of abdominal aortic aneurysm. Semin Ultrasound CT MR 31:292-300

7. Flors L, Leiva-Salinas C, Norton PT, Patrie JT, Hagspiel KD (2013) Endoleak detection after endovascular repair of thoracic aortic aneurysm using dual-source dual-energy CT: suitable scanning protocols and potential radiation dose reduction. AJR Am J Roentgenol 200:451-460

8. Macari M, Chandarana H, Schmidt B, Lee J, Lamparello P, Babb J (2006) Abdominal aortic aneurysm: Can the arterial phase at ct evaluation after endovascular repair be eliminated to reduce radiation dose? Radiology 241:908-914

9. Iezzi R, Cotroneo AR, Filippone A et al (2006) Multidetector CT in abdominal aortic aneurysm treated with endovascular repair: are unenhanced and delayed phase enhanced images effective for endoleak detection? Radiology 241:915-921

10. Brenner DJ, Elliston CD (2004) Estimated radiation risks potentially associated with full-body CT screening. Radiology 232:735-738

11. Brenner DJ, Hall EJ (2007) Computed tomography-an increasing source of radiation exposure. N Engl J Med 357:2277-2284

12. De Jong PA, Mayod JR, Golmohammadi K et al (2006) Estimation of cancer mortality associated with repetitive computed tomography scanning. Am J Respir Crit Care Med 173:199-203

13. Einstein AJ, Henzlova MJ, Rajagopalan S (2007) Estimating risk of cancer associated with radiation exposure from 64-slice computed tomography coronary angiography. JAMA 298:317-323

14. Dixon AK, Dendy P (1998) Spiral CT: how much does radiation dose matter? Lancet 352:1082-1083

15. Albrecht MH, Scholtz JE, Hüsers K et al (2016) Advanced imagebased virtual monoenergetic dual-energy CT angiography of the abdomen: optimization of kiloelectron volt settings to improve image contrast. Eur Radiol 26:1863-1870 
16. Bamberg F, Dierks A, Nikolaou K, Reiser MF, Becker CR, Johnson TRC (2011) Metal artifact reduction by dual energy computed tomography using monoenergetic extrapolation. Eur Radiol 21:1424-1429

17. Kalender WA, Schmidt B, Zank1 M, Schmidt M (1999) A PC program for estimating organ dose and effective dose values in computed tomography. Eur Radiol 9:555-562

18. Shrimpton PC, Hillier MC, Lewis MA, Dunn M (2006) National survey of doses from CT in the UK: 2003. Br J Radiol 79:968-980

19. Winklehner A, Gordic S, Lauk E, Frauenfelder T, Leschka S, Alkadhi H et al (2015) Automated attenuation-based tube voltage selection for body CTA: Performance evaluation of 192-slice dualsource CT. Eur Radiol 25:2346-2353

20. Cao P, De Rango P, Verzini F et al (2010) Endoleak after endovascular aortic repair: classification, diagnosis and management following endovascular thoracic and abdominal aortic repair. J Cardiovasc Surg (Torino) 51:53-69

21. Jouhannet C, Alsac JM, Julia P et al (2014) Reinterventions for type 2 endoleaks with enlargement of the aneurismal sac after endovascular treatment of abdominal aortic aneurysms. Ann Vasc Surg 28:192-200

22. Beitzke D, Wolf F, Edelhauser G et al (2011) Computed tomography angiography of the carotid arteries at low $\mathrm{kV}$ settings: a prospective randomised trial assessing radiation dose and diagnostic confidence. Eur Radiol 21:2434-2444

23. Habets J, Zandvoort HJ, Reitsma JB et al (2013) Magnetic resonance imaging is more sensitive than computed tomography angiography for the detection of endoleaks after endovascular abdominal aortic aneurysm repair: a systematic review. Eur J Vasc Endovasc Surg 45:340-350

24. Mori K, Saida T, Sato F, et al. Endoleak detection after endovascular aneurysm repair using unenhanced MRI with flow suppression technique: Feasibility study in comparison with contrast-enhanced CT. Eur Radiol. 2016

25. Pitton MB, Schweitzer H, Herber S et al (2005) MRI versus helical CT for endoleak detection after endovascular aneurysm repair. AJR Am J Roentgenol 185:1275-1281

26. Cejna M, Loewe C, Schoder M et al (2002) MR angiography vs CT angiography in the follow-up of nitinol stent grafts in endoluminally treated aortic aneurysms. Eur Radiol 12:2443-2450

27. Patatas K, Ling L, Dunning J, Shrivastava V (2012) Static sac size with a type II endoleak post-endovascular abdominal aortic aneurysm repair: surveillance or embolization? Interact Cardiovasc Thorac Surg 15:462-466

28. Sommer WH, Johnson TR, Becker CR et al (2009) The value of dual-energy bone removal in maximum intensity projections of lower extremity computed tomography angiography. Investig Radiol 44:285-292

29. Von Tengg-Kobligk H, Correa Londono M, Von Allmen R, Heverhagen JT, Van Den Berg JC (2014) State-of-the-art of imaging detecting endoleaks post-EVAR with special focus on low-flow endoleaks. J Cardiovasc Surg (Torino) 55:563-579

30. Cantisani V, Grazhdani H, Clevert DA et al (2015) EVAR: Benefits of CEUS for monitoring stent-graft status. Eur J Radiol 84: $1658-1665$ 